\title{
Oligosaccharide nanomedicine of alginate sodium improves therapeutic results of posterior lumbar interbody fusion with cages for degenerative lumbar disease in osteoporosis patients by downregulating serum miR-I55
}

This article was published in the following Dove Press journal:

International Journal of Nanomedicine

Number of times this article has been viewed

\section{Yang Qu \\ Zhengming Wang \\ Haohan Zhou \\ Mingyang Kang \\ Rongpeng Dong \\ Jianwu Zhao}

Department of Orthopedics, The Second Hospital of Jilin University, Changchun, People's Republic of China
Correspondence: Jianwu Zhao Department of Orthopedics, The Second Hospital of Jilin University, 218 Ziqiang Street, Changchun I3004I, People's Republic of China Tel/fax +8643188796940 Email zhao20130922@I63.com
Abstract: Degenerative lumbar disease (DLD) is a significant issue for public health. Posterior lumbar intervertebral fusion with cages (PLIFC) has high-level fusion rate and realignment on DLD. However, there are some complications following the surgery. Alginate oligosaccharides (AOS) have antioxidant and anti-inflammatory activities and may be suitable for infection therapy. MiR-155 is a biomarker associated with inflammatory and oxidative stress. AOS may promote PLIFC therapy by regulating miR-155. Pluronic nanoparticles and oligosaccharide nanomedicine of alginate sodium (ONAS) were prepared with ampicillin at size $<200 \mathrm{~nm}$. Ninety-six DLD osteoporosis patients received PLIFC and were evenly assigned into ONAS group (OG, oral administration of $100 \mathrm{mg}$ ONAS daily) and control group (PG, $100 \mathrm{mg}$ pluronic nanoparticles). Serum miR-155 level was measured by real-time quantitative PCR. The levels of superoxide dismutase (SOD), glutathione (GSH), aspartate aminotransaminase (AST), alanine aminotransferase (ALT), interleukin-1 $\beta$ (IL-1 $\beta$ ), and interleukin-1 receptor antagonist (IL-1 ra) were measured. Weighted mean difference (WMD), relative risk (RR), complications, surgery infection rate, fusion rate, and Japanese Orthopaedic Association (JOA) scores were used to evaluate therapeutic efficacy. After 1-month therapy, infection rates and side effects were lower in OG than those in PG (RR $=0.64,95 \%$ confidence interval $[\mathrm{CI}][0.48,0.84], P=0.001)$. The fusion rates were higher in $\mathrm{OG}$ than in $\mathrm{PG}(\mathrm{WMD}=21.96,95 \% \mathrm{CI}[-0.24,37.62], P=0.021)$. The JOA scores were higher in OG than in PG ( $\mathrm{RR}=0.52,95 \% \mathrm{CI}[0.33,0.84], P=0.007)$, and no significant difference was found for the visual analog scale and Oswestry Disability Index. Serum levels of miR-155, ALT, AST, and IL-1 $\beta$ were lower while SOD, GSH, and IL-1ra were higher in OG than in PG. MiR-155 mimic increased the levels of ALT, AST, and IL-1 $\beta$ and reduced the levels of SOD, GSH, and IL-1ra. In contrast, miR-155 inhibitor had reverse results. Therefore, ONAS has better improvement in complications and therapeutic effects on DLD by regulating serum miR-155.

Keywords: alginate oligosaccharide, antioxidant, anti-inflammatory, fusion rate, infection rate, Japanese Orthopaedic Association, Oswestry Disability Index

\section{Introduction}

Degenerative lumbar disease (DLD) is a significant issue for public health and may cause compression fractures and abnormal spinal curvature. Most osteoporosis patients with DLD have the experiences of back and leg pains. Furthermore, DLD leads to 
severe pain and disabilities. ${ }^{1}$ Posterior lumber interbody fusion (PLIF) as an effective method for the therapy of intervertebral disk disease has been widely accepted. Over the past decades, there were many morphological, physiological, biomechanical, and immunological researches on spine fusion. ${ }^{2}$ In many clinical cases, spinal fusion surgery is the best option to restore spinal integrity. Iliac crest bone graft has been regarded as the gold standard for the surgery to achieve spinal fusion with osteoinductive and osteoconductive functions. ${ }^{3}$ Bone grafts can be divided into allogeneic and autologous bone grafts. Generally, autologous bone graft is better than allogeneic bone graft. However, in some cases, allogeneic bone grafts may be better than autologous bone grafts, such as by reducing damaged bone area and other corresponding donor complications. These complications include infection, pain, blood loss, secondary fractures, donor instability, and neurological damage. A recent retrospective analysis demonstrates that there is no significantly statistical difference in fusion rates between allogeneic bone grafts and autologous iliac grafts. ${ }^{4}$

With the development of biomechanical research, the cage has been developed from a cylindrical form to rectangular, mesh, oval, and other forms. PLIF with cages (PLIFC) has caught much attention for its superiority in biomechanics, but high incidence of thin-layer chromatography-related complications may cause a new problem. ${ }^{5}$ In contrast, PLIF is a common choice in the treatment of osteoporosis patients with chronic DLD. The incidence and severity of complications related to cages remain the main problem in the surgery.

Alginate oligosaccharides (AOS) as natural products have beneficial effects on human health, including antioxidant ${ }^{6,7}$ and anti-inflammatory activities,${ }^{8}$ and can inhibit the development and deterioration of the disorders caused by the surgery. DLD is often associated with inflammatory status ${ }^{9}$ and oxidative stresses. ${ }^{10}$ AOS may be beneficial for preventing DLD progression. ${ }^{11,12}$ Nanotechnology has provided the possibility of delivering drugs to target tissues via nanoparticles. In this way, unwanted adverse effects can be controlled because no higher-dose medicine is needed. However, the related molecular mechanisms for the functions of oligosaccharide nanomedicine of alginate sodium (ONAS) on DLD patients remain widely unknown. MiR-155, a multifunctional microRNA, has been found to be involved with inflammatory responses by mediating various genes. ${ }^{13}$ In contrast, miR-155 deletion can reduce the generation of reactive oxygen species and oxidative stress. ${ }^{14}$ AOS may exert their antioxidant and anti-inflammatory functions by affecting the levels of miR-155. Therefore, the efficacy and safety of ONAS on the posterior lumbar interbody fusion with cages were investigated in DLD treatment by investigating the changes of serum miR-155.

\section{Methods}

\section{AOS molecular weight was determined by electrospray ionisation mass spectrometry (ESI) MASS}

AOS were purchased from Seebio Biotech (Shanghai) Co., Ltd. (Shanghai, China) and dissolved in $\mathrm{ddH}_{2} \mathrm{O}$. AOS were prepared as follows: alginate from Lonicera japonica was purchased from Shanghai Macklin Biochemical Co., Ltd. (Shanghai, China), and the mannuronic acid to guluronic acid ratio $(\mathrm{M} / \mathrm{G})$ is 2.28. Alginate lyase was prepared according to an earlier report. ${ }^{15}$ Ten grams of alginate was dissolved in $1 \mathrm{~L}$ $20 \mathrm{mM}$ Tris-HCl buffer ( $\mathrm{pH} 7.0$ ), and $10 \mathrm{U}$ alginate lyase was added. The reaction was performed at $39^{\circ} \mathrm{C}$ for $5 \mathrm{~min}$, and then, the solution was heated to $100^{\circ} \mathrm{C}$ to stop the reaction. Ethanol was added to final concentration $50 \%(\mathrm{v} / \mathrm{v})$ to remove the precipitate via centrifugation at $1,000 \times \mathrm{g}$ for $10 \mathrm{~min}$. The supernatant was filtered by a $0.45 \mu \mathrm{m}$ membrane and lyophilized. To ensure the removal of trace salt, the sample was purified by using a Micro Bio-Spin 6 Column (Bio-Rad Laboratories Inc., Hercules, CA, USA) prior to MS analysis. Samples are typically analyzed at $1 \%(\mathrm{w} / \mathrm{v})$ concentration.

MS data were obtained on a solariX FT-ICR Mass Spectrometer (Bruker Corporation, Billerica, MA, USA). In general, ionization is achieved by applying a potential of $1.4-1.8 \mathrm{kV}$ and setting the drying gas to $120^{\circ} \mathrm{C}$. Based on molecular weight of the analyzed AOS components to adjust the ion transmission radio frequency (RF) and flight time. The resulting mass spectra were externally calibrated using an ESI tuned mixture (Agilent Technologies, Santa Clara, CA, USA) and analyzed by using data analysis software (Bruker Corporation).

\section{Normal nanoparticle preparation}

Nanoparticles are typically sized in nanoscale systems at $10-1,000 \mathrm{~nm}$. These systems contain drugs by embedding them in the matrix of the particles or attaching them to the matrix of the particles. The nanocapsules are blister systems in which the drug is confined in the oily or aqueous liquid core and surrounded by the polymer film. The nanospheres are spherical particles having gelatinized internal particles in which the entrapped components are physically dispersed. Nanocapsules with structured cores have also been designed and are essentially a combination of nanocapsules and nanospheres. They are usually prepared by preparing a nanosphere and subsequently forming an additional shell on the interface of the nanosphere. 
Two grams of ampicillin was dissolved in $10 \mathrm{~mL} \mathrm{ddH}_{2} \mathrm{O}$ and mixed with pluronic-F68 $10 \mathrm{~g}$, phosphoprotein $2.0 \mathrm{~g}$, glycerol $30 \mathrm{~g}, 70 \%$ sorbitol $21 \mathrm{~g}$, and $35 \mathrm{~mL} 96 \%$ ethanol. The process can be described as follows: the phospholipid was dissolved in ethanol at room temperature. Pluronic-F68 was added and stirred at $37^{\circ} \mathrm{C}$. Ampicillin was added and stirred for $10 \mathrm{~min}$. Glycerol was added and stirred until the solution became clear. Finally, sorbitol was added and continued to stir until the solution was clear. The mixture was sterilized by $0.2 \mu \mathrm{m}$ microporous membrane, and filtered solution was lyophilized and stored at $-20^{\circ} \mathrm{C}$ freezer.

\section{ONAS preparation}

Two grams of ampicillin was dissolved in $10 \mathrm{~mL} \mathrm{ddH_{2 }} \mathrm{O}$ and mixed with AOS $10 \mathrm{~g}$, phosphoprotein $2.0 \mathrm{~g}$, glycerol $30 \mathrm{~g}$, $70 \%$ sorbitol $21 \mathrm{~g}$, and $35 \mathrm{~mL} 96 \%$ ethanol. The process can be described as follows: the phospholipid was dissolved in ethanol at room temperature. AOS were added and stirred at $37^{\circ} \mathrm{C}$. Ampicillin was added and stirred for $10 \mathrm{~min}$. Glycerol was added and stirred until the solution became clear. Finally, sorbitol was added and continued to stir until the solution was clear. The mixture was sterilized by $0.2 \mu \mathrm{m}$ microporous membrane, and filtered solution was lyophilized and stored at $-20^{\circ} \mathrm{C}$ freezer.

\section{Morphology analysis of nanoparticles by transmission electron microscopy (TEM)}

The morphology of nanoparticles was observed by using a Philips CM12 TEM (FEI, Hilsboro, OR, USA) with $100 \mathrm{kV}$ accelerating voltage. One drop of the suspension diluted with water was placed on a carbon-coated copper grid and dried in the air before observation.

\section{The effects of AOS toxicity on MG-63 human osteosarcoma cells}

MG-63 human osteosarcoma cells were purchased from the Cell Bank of Chinese Academy of Sciences (Shanghai, China) and cultured in DMEM with 10\% fetal bovine serum (FBS) at $37^{\circ} \mathrm{C}$ and $5 \% \mathrm{CO}_{2}$. The cell monolayer was digested by trypsin when the cells grew up to $90 \%$ confluency. The cells were washed with fresh DMEM three times and adjusted to $1 \times 10^{6}$ cells $/ \mathrm{mL}$. Different concentrations $(0,0.1,1,10,100$, and $1,000 \mu \mathrm{g} / \mathrm{mL})$ of AOS were added to MG-63 human osteosarcoma cells and further cultured for 3 days. Trypan blue was used to measure live cells under a microscope. Meanwhile, $\left[{ }^{3} \mathrm{H}\right]$ thymidine absorption was used to measure the activity of the cells. Two hundred microliters of cell suspension of $1.0 \times 10^{5}$ cells $/ \mathrm{mL}$ was added to each well of a 96-well plate. After 1-day culture, cells were labeled with $1 \mathrm{Ci} /$ well of $\left[{ }^{3} \mathrm{H}\right]$ thymidine for $3 \mathrm{~h}$. Absorption values were measured with a liquid scintillation counter (model SN 6930; Shanghai Hesuo Rihuan Photoelectric Instrument Co., Shanghai, China).

\section{Cell transient transfection}

Different concentrations $(0,0.1,1,10$, and $100 \mu \mathrm{g} / \mathrm{mL})$ of AOS were added to cells and further cultured for 3 days under the same condition. The level of miR-155 was measured. The cells were transfected with miR-155 mimic or inhibitors (single-stranded chemically modified oligonucleotides; Shanghai GenePharma Co., Ltd., Shanghai, China) by using Lipofectamine $^{\circledR} 2000$ (Thermo Fisher Scientific, Waltham, MA, USA).

\section{Patient screening}

Before the experiment, all procedures were approved by the Ethics Committee of The Second Hospital of Jilin University (approval no 20151213002N). Written informed consent was obtained from each patient with his/her signed name. The following inclusion criteria were used: 1) prospective, randomized, and/or nonrandomized controlled studies for DLD therapy; 2) the DLD osteoporosis patients had the symptoms of lumbar spinal stenosis, instability and spondylolisthesis (I and II degrees), and lumbar disk herniation; 3) the osteoporosis patients had low back pain, intermittent claudication, lumbar spinal canal decompression, and persistent lower extremity symptoms; 4) the osteoporosis patients with DLD were confirmed by auxiliary examination (X-ray, $\mathrm{CT}$, and MRI).

The following exclusion criteria were used: 1) the osteoporosis patients had severe intervertebral space collapse with peripheral osteophyte formation; 2) the osteoporosis patients had lumbar degenerative scoliosis (Cobb angle $>10^{\circ}$ ); 3) the osteoporosis patients suffered from osteoporosis; 4) the osteoporosis patients suffered from diabetes; 5) the osteoporosis patients had serious medical diseases and contraindications; and 6) the osteoporosis patients had mental disorders.

\section{Patient grouping}

Based on the inclusion and exclusion criteria, 96 DLD patients were recruited from May 12, 2014, to February 14, 2015. All the patients received PLIFC from March 3-13, 2016, at our hospital. After surgery, all patients were evenly assigned into ONAS group (OG, daily oral administration of $500 \mathrm{mg}$ ONAS) and control group (PG, daily oral administration of $500 \mathrm{mg}$ pluronic nanoparticles). 


\section{Blood nanoparticle analysis}

A blood sample was collected from each patient in tubes containing EDTA anticoagulant. Blood smears were prepared immediately from samples taken with anticoagulant. The blood smear was made on clean glass slides and observed under a light microscope (Olympus, Tokyo, Japan) at 100× magnification under oil immersion.

\section{Data extraction and analysis}

The following parameters were measured and analyzed: the number of osteoporosis patients, sex ratio, patient age, follow-up time, patient diagnosis, surgical segments, the number of osteoporosis patients who received cage fusion, intraoperative blood loss, the duration of surgery and hospital stay, preoperative and prospective visual analog scale (VAS), Japanese Orthopaedic Association (JOA), and Oswestry Disability Index (ODI) scores, fusion rates, and complication rates. Data were processed by RevMan 5.3 (Cochrane Collaboration, Oxford, UK). The data were collected and analyzed quantitatively. For the continuity index, the deviation was pooled into weighted mean difference (WMD) and the $95 \%$ confidence interval (CI) was used for the statistics. For the discontinuity index, the odds ratio risk, relative risk (RR), and 95\% CI were used to evaluate the dichotomous results. The heterogeneity of the included studies was judged by the $P$-value, and the heterogeneity was judged according to $I^{2}$ value. If $P>0.1, I^{2}<0.5$, they could be considered homogeneous with similar studies and a fixed-effect model would be used for meta-analysis; if $P<0.1, P \geq 0.5$, a random-effect model would be selected. If there was heterogeneity between the studies, the original data and extraction methods would be reanalyzed. A descriptive method would be considered if the data could not be analyzed by using the two methods.

\section{Heterogeneity analysis}

The sources of heterogeneity were identified and examined in sub-group and sensitivity analyses. Heterogeneity was evaluated by using $I^{2}$ values, of which $<25 \%$ was regarded as low heterogeneity, $50 \%$ was regarded as mild, and $75 \%$ was regarded as high. If the effect of the merger between the patients with cage fusion and the patients without cage fusion was decreased significantly, $P<0.1$, and the heterogeneity could not be determined.

\section{Quantitative RT-PCR analysis}

Blood samples were obtained from DLD patients, and serum was purified within $1 \mathrm{~h}$. Total RNA was isolated by using an RNA purification kit (TRI Reagent ${ }^{\circledR}$; Molecular Research
Center, Cincinnati, OH, USA). The level of miR-155 was normalized to total RNA. qRT-PCR was carried out on a 7500 Real-Time PCR System (Thermo Fisher Scientific) by using a universal reaction condition: $95^{\circ} \mathrm{C}$ for $2 \mathrm{~min}$, followed by 40 cycles of $95^{\circ} \mathrm{C}$ for $10 \mathrm{~s}$ and $68^{\circ} \mathrm{C}$ for $30 \mathrm{~s}$. After the PCR, $C_{\mathrm{T}}$ values were calculated and expressed by using $2^{-\Delta \mathrm{G}}$.

\section{Biochemical analysis}

The serum activity of superoxide dismutase (SOD) was measured by formazan-WST method. The serum concentration of reduced glutathione (GSH) was determined by the dithiobis-2nitrobenzoic acid method. The serum concentrations of aspartate aminotransaminase (AST) and alanine aminotransferase (ALT) were evaluated by biochemical analyzer (Fenghui Co., Ltd., Shanghai, China). The levels of IL-1 $\beta$ and IL-1 ra were measured by using ELISA kits (catologue numbers ab46052 and ab174450) from Abcam (Shanghai, China).

\section{Statistical analysis}

Student's $t$-test and chi-squared statistic tests were performed using SPSS Version 20.0. The differences were considered statistically significant if $P<0.05$.

\section{Results \\ Characterization of ONAS}

The main components of AOS (DP20 to DP35) were confirmed by ESI MS under the conditions that produced mass spectra with $\mathrm{M}+\mathrm{H}^{+}$. Figure 1 shows that the masses predicted from DP20 (C120H142O121Na20) to DP35 (C210H247O122Na35) are 4,176 and 7,146 Da, respectively.

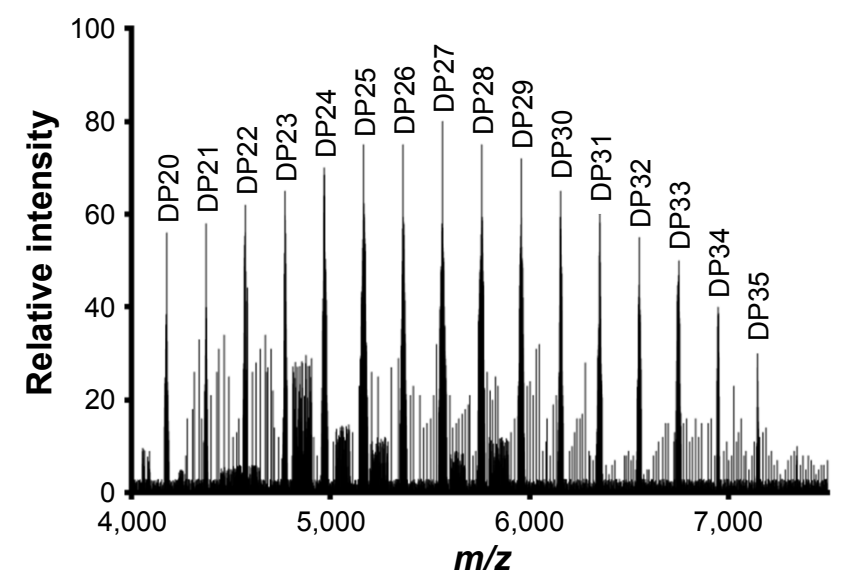

Figure I ESI mass spectrometry analysis of bioactive fractions from ONAS under the conditions that produced mass spectra with $\mathrm{M}+\mathrm{H}^{+}$.

Note: Mass spectra were visualized following the separation of DP20 $\left(\mathrm{M}+\mathrm{H}^{+}=\right.$ 4, I77 Da) to DP35 $\left(\mathrm{M}+\mathrm{H}^{+}=7,147 \mathrm{Da}\right)$.

Abbreviations: ESI, electrospray ionisation; ONAS, oligosaccharide nanomedicine of alginate sodium. 


\section{Morphological characterization of nanoparticles}

The TEM was used to characterize the morphology of the nanoparticles. Figure 2 shows the TEM images for two samples of nanoparticles obtained by using pluronic-F68 (Figure 2A) or AOS (Figure 2B). All nanoparticles are in tube form. These images show that the particle size of nanoparticles is $<200 \mathrm{~nm}$. Light microscope images revealed that blood cells were regularly spherical in shape and had a generally smooth surface with an average size of $\sim 10 \mu \mathrm{m}$. Nanoparticles could be observed in the patients with pluronic nanoparticles (Figure 2D) or ONAS nanoparticles (Figure 2E) when compared with a control without nanoparticles (Figure 2C).

\section{The effects of AOS toxicity on MG-63 human osteosarcoma cells}

To avoid the effects of AOS toxicity on MG-63 human osteosarcoma cells, a suitable concentration of AOS was determined. Trypan blue analysis indicated that exposure of the MG-63 cells to AOS, in the presence of $>100 \mu \mathrm{g} / \mathrm{mL}$, resulted in the loss of cell viability (Figure $3 \mathrm{~A}, P<0.05$ ). The toxicity of AOS was determined via $\left[{ }^{3} \mathrm{H}\right]$ thymidine incorporation into DNA after exposure to different concentrations of AOS.
There was significant reduction in $\left[{ }^{3} \mathrm{H}\right]$ thymidine uptake when the concentration was $>100 \mu \mathrm{g} / \mathrm{mL}$ (Figure $3 \mathrm{~B}, P<0.05$ ). Based on these results, we assessed that administration of $100 \mathrm{mg}$ AOS daily will not cause toxicity to human cells.

\section{AOS reduce miR-I 55 level in MG-63 human osteosarcoma cells}

qRT-PCR analysis showed that AOS reduce miR-155 level in MG-63 human osteosarcoma cells when the concentration was $>1 \mu \mathrm{g} / \mathrm{mL}$ (Figure 4). Meanwhile, the level of miR-155 was further reduced with the increase in the AOS concentration (Figure 4). The results suggest that AOS reduce miR-155 level in MG-63 human osteosarcoma cells.

\section{MiR-I55 increase oxidant stress of MG-63 human osteosarcoma cells}

Table 1 shows that miR-155 mimics reduced antioxidant functions by inhibiting the activities of SOD and GSH in MG-63 cells and increasing the levels of ALT $(P<0.05)$. In contrast, miR-155 inhibitor increased antioxidant functions by improving the activities of SOD and GSH in MG-63 cells and reducing the levels of ALT $(P<0.05)$. The results suggest that miR-155 increases oxidant stress of MG-63 cells.

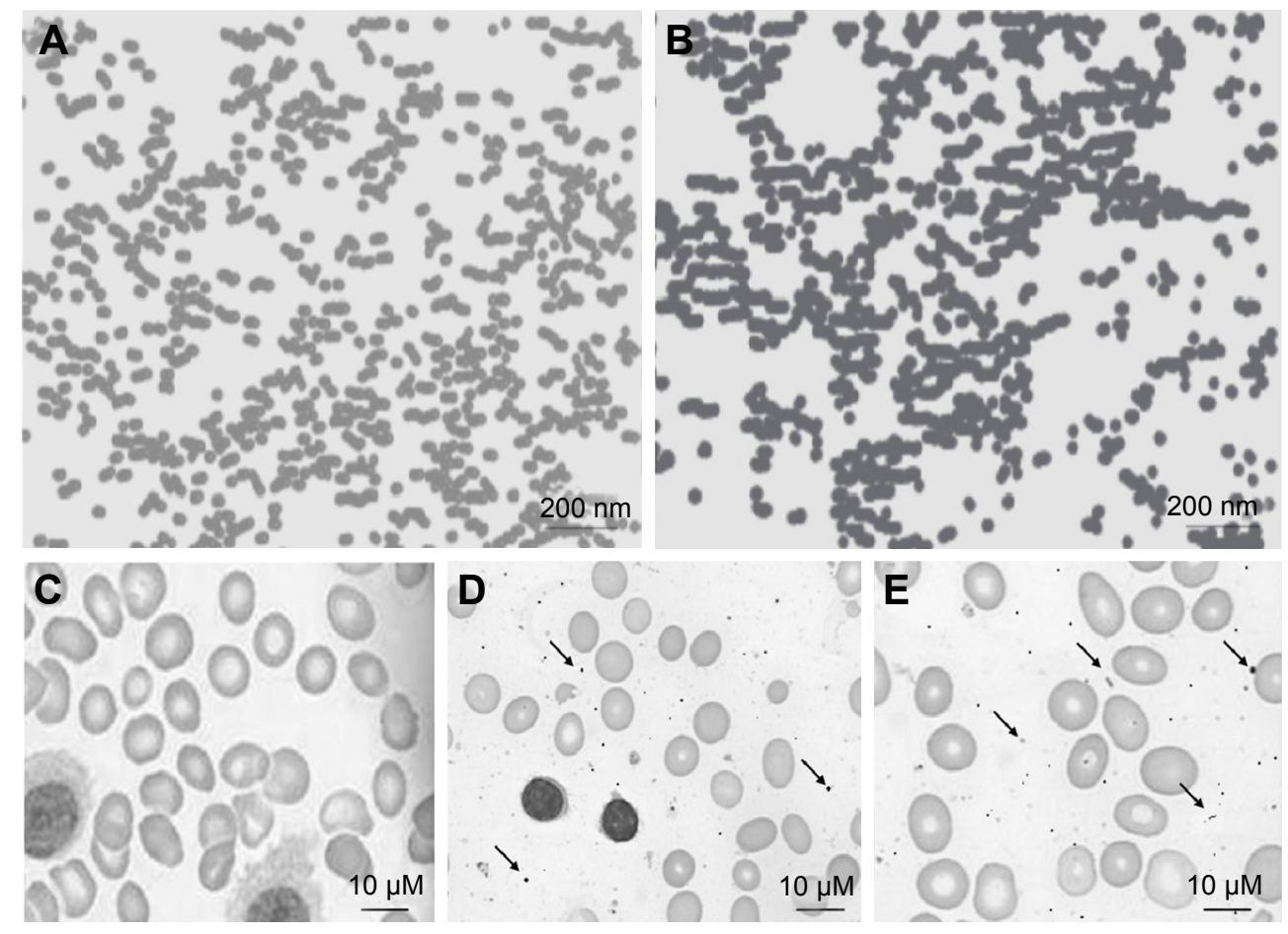

Figure 2 Images of nanoparticles.

Notes: (A) TEM images of pluronic nanoparticles. (B) TEM images of AOS nanoparticles. (C) Light micrograph of blood cells in a human blood smear without nanoparticles. (D) Light micrograph of blood cells in a human blood smear with pluronic nanoparticles. (E) Light micrograph of blood cells in a human blood smear with AOS nanoparticles. Arrows show the nanoparticles in blood samples.

Abbreviations: AOS, alginate oligosaccharides; TEM, transmission electron microscopy. 

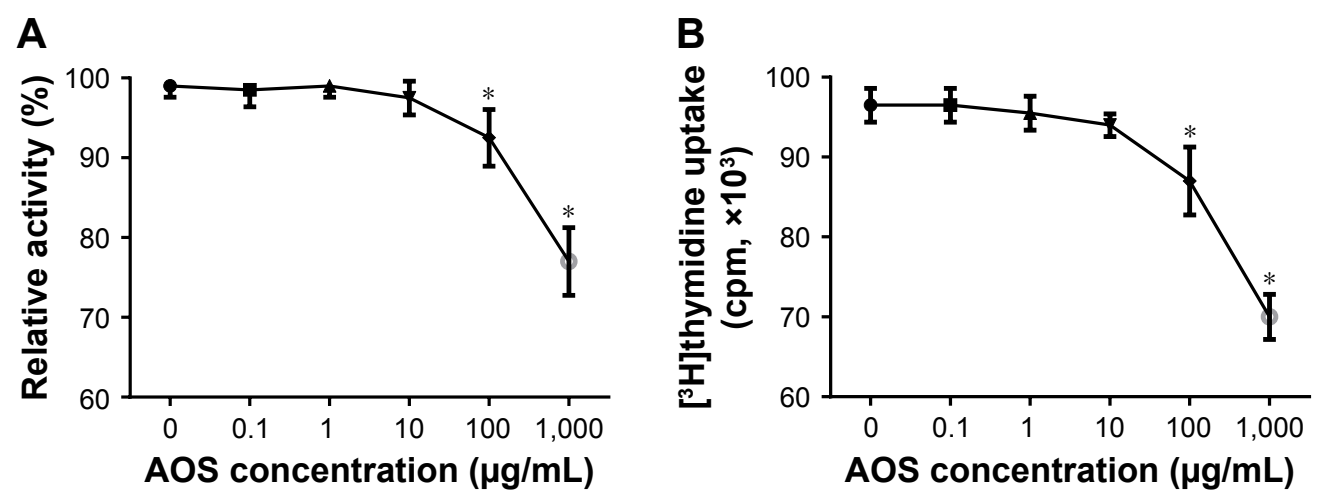

Figure 3 The effects of AOS on the viability of MG-63 cells.

Notes: (A) Cell viability of MG-63 was measured using Trypan blue. (B) Cell viability of MG-63 was measured using $\left[{ }^{3} \mathrm{H}\right]$ thymidine uptake. The data are presented as the mean \pm standard deviation. $n=5$ in each group.

Abbreviation: AOS, alginate oligosaccharides.

\section{MiR-I55 increases inflammatory activities of MG-63 human osteosarcoma cells}

ELISA analysis showed that there was no statistically significant difference for serum levels of IL-1 $\beta$ (Figure 5A, $P>0.05$ ) and IL-1ra (Figure 5B, $P>0.05$ ) on 0 -day culture. After 3-day culture, miR-155 mimics increase the serum levels of IL-1 $\beta$ (Figure 6A, $P<0.05$ ) and reduce the levels of IL-1 ra (Figure 5B, $P<0.05$ ). In contrast, miR-155 inhibitors decrease the serum levels of IL- $1 \beta$ (Figure $5 \mathrm{~A}, P<0.05$ ) and increase the serum levels of IL-1ra (Figure 5B, $P<0.05$ ). All the results suggest that miR-155 increases inflammatory activities of MG-63 human osteosarcoma cells.

\section{Baseline clinical characterization of patients}

A total of 96 patients were recruited, including 59 males and 37 females. All patients underwent PLIFC. Baseline characteristics of patients in the study are shown in Table 2.

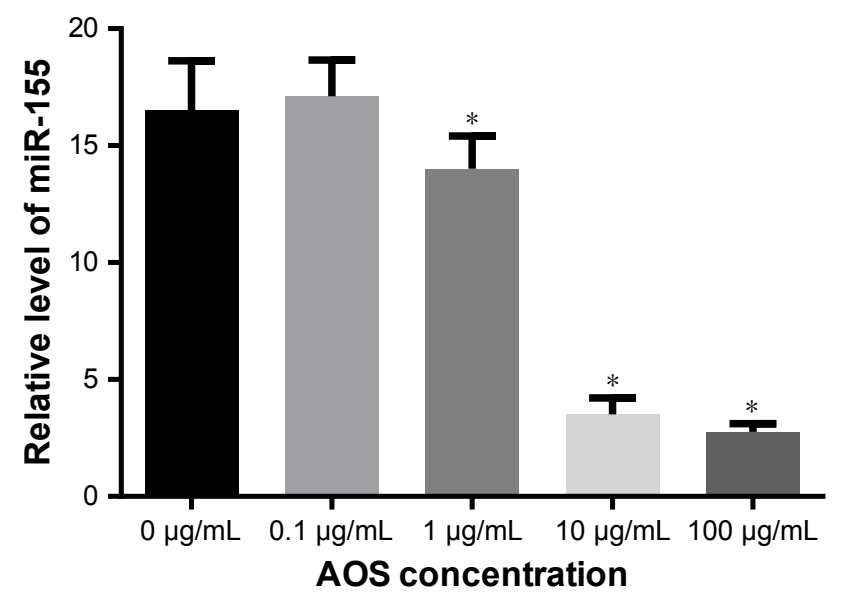

Figure 4 The effects of AOS on the level of miR-155 in MG-63 human osteosarcoma cells.

Notes: ${ }^{*} P<0.05$ via a control group without AOS addition. There are significantly statistical differences if $P<0.05$.

Abbreviation: AOS, alginate oligosaccharides.
There was no significant difference in age between the two groups of osteoporosis patients with degenerative lumbar disease at mean age 32.4 years (range 24.1-40.0 years). There was no pseudarthrosis, instrumentation failures, or significant subsidence during the surgery. Disk height was increased from $5.3 \pm 2.1 \mathrm{~mm}$ before surgery to $9.5 \pm 3.1 \mathrm{~mm}$ after surgery. The mean operative time was $142 \pm 34 \mathrm{~min}$. The mean blood loss was $716 \pm 326 \mathrm{~mL}$ in all patients. There was no obvious difference in the baseline characters for preoperative symptoms between the two groups $(P>0.05)$. There were significant differences in the postoperative symptoms, infection, and back pain between the two groups $(P<0.05)$.

\section{Integrated analysis of clinical efficacy}

The main curative efficacy was evaluated by using VAS, JOA, and ODI scores, complications, and fusion rate.

The VAS score was used to assess the pain levels. There was no statistically significant difference in the VAS scores between the two groups in a fixed-effect model before surgery $(\mathrm{WMD}=-0.08,95 \% \mathrm{CI}[-0.39,0.23], P=0.63)$. There was no statistically significant difference for VAS score in a random-effect model between the two groups at the final follow-up examination after surgery $(\mathrm{WMD}=0.22,95 \% \mathrm{CI}$ $[-2.49,2.93], P=0.87)$.

The JOA score was used to evaluate the functional recovery of lumbar vertebrae. There was no statistically significant difference in the JOA score between the two groups before surgery $(\mathrm{WMD}=0.29,95 \% \mathrm{CI}[-0.31,0.89], P=0.35)$. OG showed better improvement when compared with PG after surgery $(\mathrm{RR}=0.52,95 \% \mathrm{CI}[0.33,0.84], P=0.007)$.

The complication rates were similar in a fixed-effect model between the two groups before surgery $(\mathrm{RR}=0.85,95 \%$ CI $[0.64,1.13], P=0.26)$. The fusion rates of the lumbar vertebrae were evaluated in final follow-up examination 
Table I The effects of miR-I55 on the antioxidant activities in MG-63 human osteosarcoma cells

\begin{tabular}{lllll}
\hline Group & SOD $(\mathbf{U} / \mathrm{mL})$ & GSH $(\mathbf{n g} / \mathbf{L})$ & ALT $(\mathbf{U} / \mathbf{m L})$ & AST $(\mathbf{U} / \mathbf{m L})$ \\
\hline Control & $21.36 \pm 3.17$ & $20.78 \pm 2.35$ & $40.93 \pm 4.57$ & $83.25 \pm 11.36$ \\
MiR-I55 mimic & $10.23 \pm 3.29^{*}$ & $8.17 \pm 1.58^{*}$ & $67.39 \pm 9.75^{*}$ & $84.33 \pm 15.28$ \\
MiR-I55 inhibitor & $46.13 \pm 6.48^{*}$ & $42.36 \pm 9.36^{*}$ & $26.99 \pm 8.74^{*}$ & $86.78 \pm I 1.49$ \\
\hline
\end{tabular}

Notes: Eight blood samples were analyzed in each group. All data are presented as mean value $\pm S D$. $* P<0.05$ vs a control group.

Abbreviations: ALT, alanine aminotransferase; AST, aspartate aminotransaminase; GSH, glutathione; SOD, superoxide dismutase.

after surgery. The fusion rates were higher in $\mathrm{OG}$ than in PG $(\mathrm{RR}=0.64,95 \%$ CI $[0.48,0.84], P=0.001)$.

The secondary clinical outcomes were evaluated by using surgical duration, the amounts of intraoperative blood loss, height of intervertebral space, ODI score, and abnormal movement of lumbar segment. The surgical duration was similar between the two groups (Table 2). There was no statistically significant difference between the two groups in a random-effect model (WMD $=14.69,95 \%$ CI $[-8.24,37.62]$, $P=0.21)$. There was no statistically significant difference for the quantity of blood loss between groups in a randomeffect model (WMD $=21.96,95 \%$ CI [-67.44, 111.36], $P=0.63)$. There was a statistically significant difference for the increase of the height of intervertebral disk between the two groups in a random-effect model (WMD $=1.31,95 \% \mathrm{CI}$ $[0.78,1.85], P<0.001)$.

ODI score was used to evaluate the functional recovery of the lumbar vertebrae. Three studies reported ODI score before surgery and at the final follow-up examination after surgery. There was no statistically significant difference in the ODI score in a fixed-effect model between the two groups (WMD $=0.99,95 \%$ CI $[-0.48,2.46], P=0.19$ ). There was no statistically significant difference for ODI score in the random-effect model between the two groups at the final follow-up examination after surgery (WMD $=2.52,95 \% \mathrm{CI}$ $[-2.26,7.29], P=0.30)$.

There was no statistically significant difference for abnormal movement of lumbar segment between the two groups in a fixed-effect model (WMD $=-0.37,95 \%$ CI $[-1.96,1.22]$, $P=0.65$ ). Similarly, there was no statistically significant difference for abnormal movement of lumbar segment between the two groups in a random-effect model (WMD $=-0.05$, $95 \%$ CI $[-1.1,0.02], P=0.16)$.

\section{Quality assessment of included studies}

Methodological Index for Non-Randomized Studies (MINORS) was used to assess bias risk of nonrandomized trials and bias risk profile. Furthermore, the comparison was performed before and after surgery for all the osteoporosis patients. All studies had low-risk publication and attrition bias. The Grading of Recommendations Assessment, Development and Evaluation (GRADE) system was used to evaluate the quality of the evidence, and the results had an average MINORS score of 15.81. Prospective studies and randomized control trials were included, and the related parameters (coherence in osteoporosis patients, key indicators, adequate
A

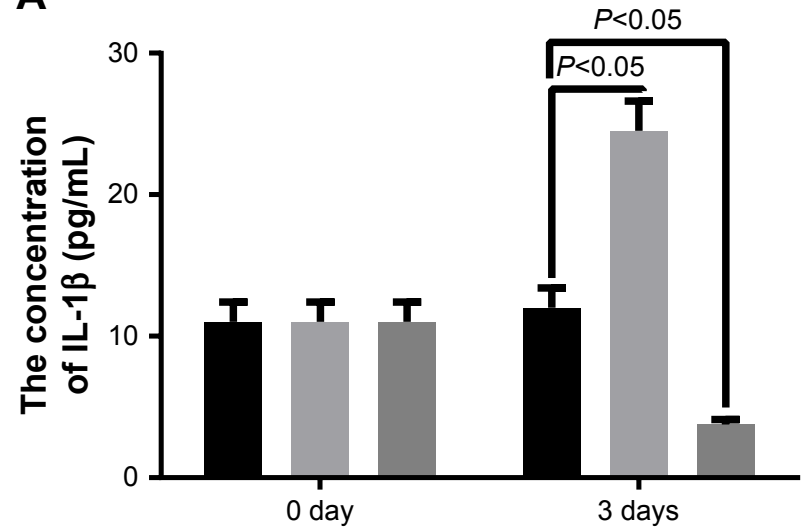

B

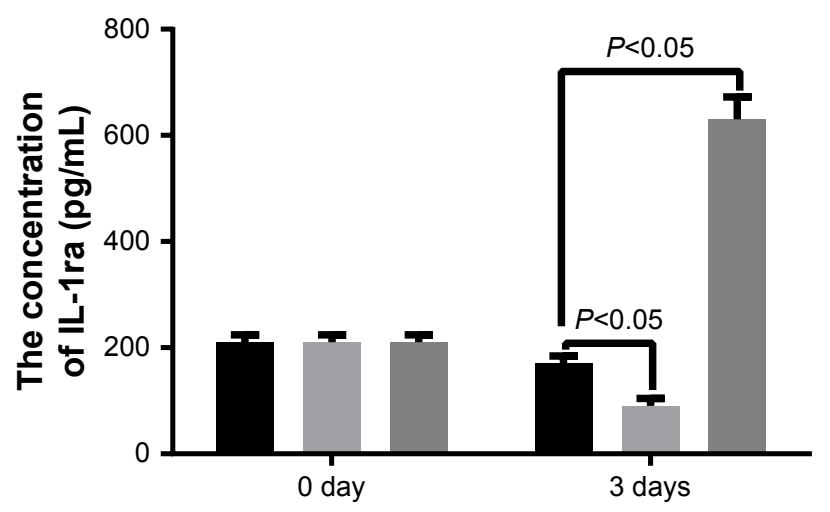

Control miR-155 mimics miR-155 inhibitors

Figure 5 The effects of miR-I55 on the anti-inflammatory activities in MG-63 human osteosarcoma cells.

Notes: (A) The effects of miR-I55 on the level of IL-I $\beta$ in MG-63 cells. (B) The effects of miR-I55 on the level of IL-Ira in MG-63 cells. There are significant statistical differences if $P<0.05$ vs a control group.

Abbreviations: IL-I $\beta$, interleukin-I $\beta$; IL-I ra, interleukin-I receptor antagonist. 


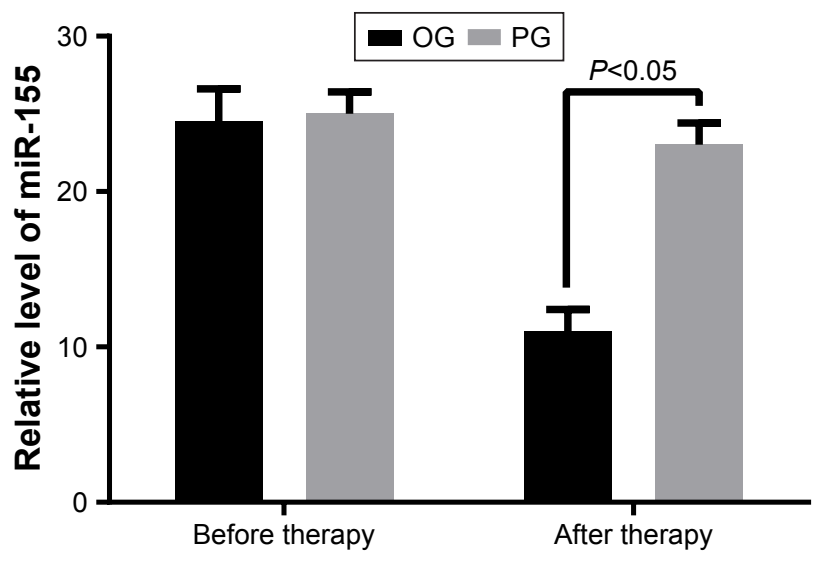

Figure 6 The effects of ONAS on the level of miR-I55 in DLD patients. Notes: ONAS group (OG, daily oral administration of $100 \mathrm{mg}$ ONAS) and control group (PG, daily oral administration of $100 \mathrm{mg}$ pluronic nanoparticles). There are significant statistical differences between $O G$ and $P G$ groups if $P<0.05$.

Abbreviations: DLD, degenerative lumbar disease; ONAS, oligosaccharide nanomedicine of alginate sodium.

follow-up time, follow-up rate, sample size, control group, baseline characters, and statistical analysis) were investigated. The results demonstrated that most studies were with low-risk bias.

\section{Serum level of miR-I 55 was higher in PG than in $O G$}

qRT-PCR analysis showed that there was no significant statistical difference for serum level of miR-155 between

Table 2 Baseline characters of all degnerative lumbar disease patients

\begin{tabular}{|c|c|c|c|}
\hline Characteristics & $\begin{array}{l}\text { OG } \\
(n=48)\end{array}$ & $\begin{array}{l}P G \\
(n=48)\end{array}$ & P-values \\
\hline Male (\%) & $29(58.3)$ & $31(64.6)$ & $0.67^{a}$ \\
\hline Mean age, years (SD) & $33.2(6.8)$ & $31.5(7.4)$ & $0.08^{\mathrm{b}}$ \\
\hline Operative time, $\min (\mathrm{SD})$ & $140(30)$ & $146(36)$ & $0.26^{b}$ \\
\hline Blood loss, mL (SD) & $712(32 I)$ & $735(328)$ & $0.49^{b}$ \\
\hline Disk height increase, mm (SD) & $4.1(1.8)$ & $3.9(2.1)$ & $0.17^{b}$ \\
\hline \multicolumn{4}{|l|}{ Preoperative symptoms, n (\%) } \\
\hline Dyspnea, n (\%) & $18(37.5)$ & $16(33.3)$ & $0.67^{\mathrm{a}}$ \\
\hline Back pain, n (\%) & $20(4 I .6)$ & $21(43.8)$ & $0.84^{\mathrm{a}}$ \\
\hline Fatigue, decreased energy, n (\%) & II (22.9) & $9(18.8)$ & $0.62^{\mathrm{a}}$ \\
\hline Tachycardia/palpitations, n (\%) & $6(12.5)$ & $7(14.6)$ & $0.77^{a}$ \\
\hline Dizziness, n (\%) & $2(4.2)$ & $3(6.3)$ & $1.00^{\mathrm{a}}$ \\
\hline Syncope, n (\%) & $3(6.3)$ & $2(4.2)$ & $1.00^{\mathrm{a}}$ \\
\hline Dysphagia, n (\%) & $3(6.3)$ & $3(6.3)$ & $1.00^{\mathrm{a}}$ \\
\hline \multicolumn{4}{|l|}{ Postoperative complications, n (\%) } \\
\hline Surgery reintervention, $\mathrm{n}(\%)$ & $0(0.0)$ & $5(10.4)$ & $0.07^{\mathrm{a}}$ \\
\hline Surgery infection, n (\%) & $0(0.0)$ & $6(12.5)$ & $0.04^{\mathrm{a}}$ \\
\hline Chronic wound infection, n (\%) & I (2.I) & $8(16.7)$ & $0.04^{a}$ \\
\hline Prolonged pain, n (\%) & I (2.I) & $9(18.8)$ & $0.02^{\mathrm{a}}$ \\
\hline
\end{tabular}

Notes: There are significantly statistical differences if $P<0.05$. ${ }^{a}$ Chi-squared test. 'Student's $t$-test.

Abbreviations: OG, alginate oligosaccharide treated; PG, placebo treated.
OG and PG groups before nanoparticle treatment $(P=0.128)$ (Figure 6). After 1-month therapy, serum level of miR-155 was higher in $\mathrm{PG}$ than in $\mathrm{OG}(P=0.023)$ (Figure 6). Some serum microRNAs have poor stability. According to the results shown in Figure 5, miR-155 was the same in a control group after 1 month $(P>0.05)$. The result suggests that miR-155 was stable within one month.

\section{Antioxidant activities were higher in $O G$ than in PG}

Before nanoparticle treatment, there was no significant statistical difference for antioxidant activities between OG and PG groups according to activities of SOD, GSH, ALT and AST (Table 3, $P>0.05$ ). After 1-month therapy, serum levels of SOD and GSH were higher in OG than in PG (Table 3, $P<0.05$ ) while serum levels of ALT were lower in OG than in PG (Table 3, $P<0.05$ ). The results suggest that AOS treatment increases antioxidant activities of DLD patients.

\section{Anti-inflammatory activities were higher in $O G$ than in PG}

ELISA analysis showed that there was no significant statistical difference for serum levels of IL- $1 \beta$ and IL-1 ra between OG and PG groups before nanoparticle treatment $(P>0.05)$ (Figure 7). After 1-month therapy, serum level of IL-1 $\beta$ was higher in $\mathrm{PG}$ than in OG $(P<0.05)$ (Figure $7 \mathrm{~A})$, while serum level of IL-1 ra was lower in PG than in OG $(P<0.05)$ (Figure 7B). The results suggest that AOS treatment increases anti-inflammatory activities of DLD patients.

\section{Discussion}

PLIF has been proposed for many decades, applied to clinical trials, and developed into basic techniques for spine surgery. Cage fusion belongs to the interfacial fixation technique. Some articles report that the disadvantages of cage fusion are with long surgical duration, more hemorrhage, and nerve root injury. ${ }^{16}$ Each method has advantages and disadvantages, and the value of PLIFC still needs to be investigated. Therefore, we compared 2,138 osteoporosis patients undergoing PLIFC, and 1,037 osteoporosis patients undergoing PLIF without cages to investigate the efficacy and safety of PLIFC and without cages in DLD treatment.

VAS, JOA, and ODI scores were used to evaluate clinical outcomes of each method. There was no statistically significant difference for these scores in a fixed-effect model between the two groups before surgery and immediate examination after surgery. After the merger collected data from literature, the difference was statistically significant 
Table 3 Biochemical parameters of enzyme activities for DLD patients

\begin{tabular}{llllll}
\hline & Group & SOD $(\mathbf{U} / \mathbf{m L})$ & GSH $(\mathbf{n g} / \mathbf{L})$ & ALT (U/mL) & AST (U/mL) \\
\hline Before therapy & OG & $27.24 \pm 3.26$ & $24.15 \pm 2.14$ & $45.12 \pm 10.43$ & $103.32 \pm 25.17$ \\
& PG & $26.25 \pm 4.16$ & $23.23 \pm 1.93$ & $47.79 \pm 8.40$ & $108.26 \pm 19.64$ \\
After therapy & OG & $32.34 \pm 5.34^{*}$ & $34.38 \pm 8.02^{*}$ & $31.45 \pm 12.18^{*}$ & $106.19 \pm 25.28$ \\
& PG & $27.34 \pm 2.32$ & $24.28 \pm 1.74$ & $48.79 \pm 12.36$ & $109.42 \pm 24.48$ \\
\hline
\end{tabular}

Notes: Eight blood samples were analyzed in each group. All data are presented as mean value $\pm S D$. *P $<0.05$ vs $P G$ group.

Abbreviations: ALT, alanine aminotransferase; AST, aspartate aminotransaminase; DLD, degenerative lumbar disease; GSH, glutathione; OG, alginate oligosaccharide treated; PG, placebo treated; SOD, superoxide dismutase.

in a fixed-effect model between the two groups $(\mathrm{RR}=0.52$, $95 \%$ CI $[0.33,0.84], P=0.007)$. There was no heterogeneity since $I^{2}=34 \%$. The results of the merger fell on the left side of the invalid line, indicating that AOS had better therapeutic results in the PLIFC.

Although the small number of samples, the heterogeneity of the study was not a reason, the results had clinical significance. Fusion rate has become one of the controversy issues in the PLIFC fusions. ${ }^{17,18}$ There were statistically significant differences for fusion rates in a fixed-effect model between the two groups at final follow-up examination after surgery $(\mathrm{RR}=0.64$, $95 \% \mathrm{CI}[0.48,0.84], P=0.001)$. There was no heterogeneity in the selected articles, and the results of the merger fell on the left side of the invalid line. Therefore, the group undergoing the ONAS had a higher fusion rate than the PG group.

Surgical duration is an important quality metric for spinal fusion, and decreased surgical time will be beneficial for improving patient outcomes. Here, the present study was used to compare the surgical duration. The results demonstrated that the surgical duration was similar between the two groups in a random-effect model (WMD $=14.69,95 \%$ CI $[-8.24$, 37.62], $P=0.21)$. The heterogeneity of the combined results was not significant.
Fusion surgery is the main method for the therapy of DLD, but the method is often accompanied with substantial blood loss. We investigated the differences of surgical blood loss, in the osteoporosis patients undergoing the surgery for DLD, between groups. There was no statistically significant difference for blood loss between the two groups (WMD $=21.96,95 \%$ CI $[-67.44,111.36], P=0.63)$.

For both groups, the abnormal motion of the surgical segment was similar in a fixed-effect model between the two groups before surgery and at the final follow-up examination after surgery. Abnormal motion may be caused by physiological loads, and thus, it is not associated with an obvious increase in motion. In statistical analysis, abnormal motion is often followed by non-Gaussian heavy-tailed behavior and other activities. It may take a long time to stimulate abnormal motion.

Alginate particles have been reported to have antioxidant activities, ${ }^{19,20}$ but the related molecular mechanisms remain unknown. AOS have antioxidant and anti-inflammatory activities and may be suitable for infection therapy. Thus, we want to find the microRNA associated with oxidative stress and inflammation. In a review paper, $>10$ microRNAs have been reported to regulate oxidative stress and/or inflammation. ${ }^{21}$
A

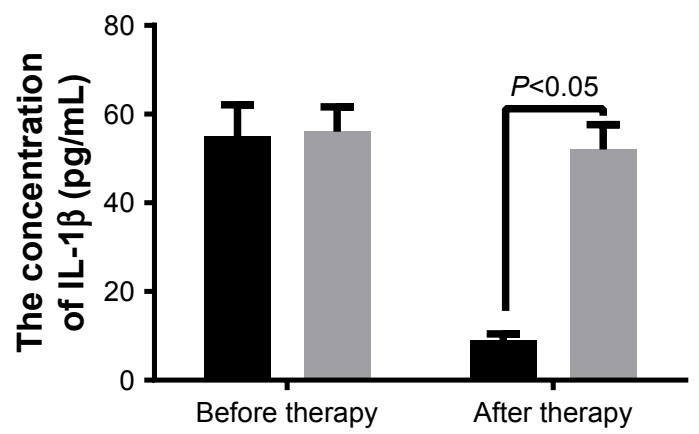

B

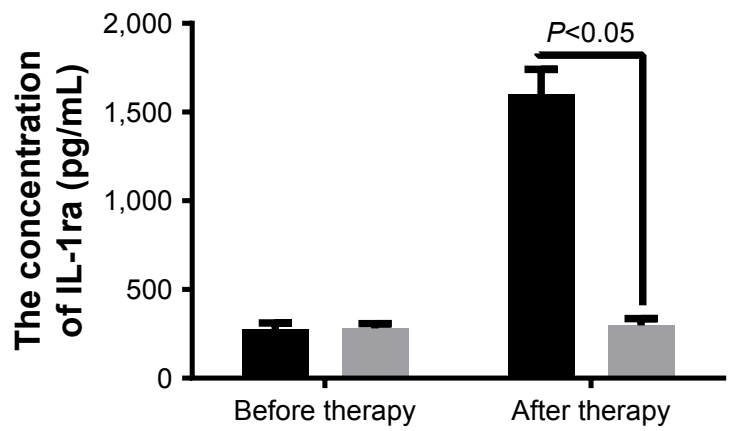

Figure 7 The effects of ONAS on the level of cytokines in DLD patients.

Notes: ONAS group (OG, daily oral administration of $100 \mathrm{mg}$ ONAS) and control group (PG, daily oral administration of 100 mg pluronic nanoparticles). (A) The effects of ONAS on the level of IL-I $\beta$ in DLD patients. (B) The effects of ONAS on the level of IL-I ra in DLD patients. There are significant statistical differences between OG and $P G$ groups if $P<0.05$.

Abbreviations: DLD, degenerative lumbar disease; IL-I $\beta$, interleukin-I $\beta$; IL-I ra, interleukin-I receptor antagonist; ONAS, oligosaccharide nanomedicine of alginate sodium. 
However, only miR-155 has been clearly reported to be associated with both oxidative stress and inflammation. ${ }^{22}$ Thus, miR-155 may be a potential target of AOS and was chosen in the present study. Here, we find that AOS can increase the antioxidant activities of DLD patients by downregulating the serum levels of miR-155 (Figure 4 and Table 2). Meanwhile, miR-155 is a main factor for inducing inflammatory responses. ${ }^{23,24}$ Thus, AOS also increases antiinflammatory properties of DLD patients by controlling miR-155 level. Hence, all microRNA should be screened in future work to confirm the present results.

ONAS is mainly made of AOS, which have a mild reaction condition and excellent gel properties and are easy to purify. AOS are environmentally friendly, $\mathrm{pH}$ neutral, and biodegradable with fewer side effects for human health. More importantly, AOS are made from alginate, which has abundant supplies in nature. Thus, AOS can be used world wide. The main difference between $\mathrm{OG}$ and $\mathrm{PG}$ is that AOS are used in OG and pluronics are used in PG. Both nanomaterials have sparked a considerable interest as vehicles used for therapeutic agents. Pluronic block copolymers affect cellular functions such as mitochondrial respiration, ATP synthesis, apoptosis, and gene expression. ${ }^{25}$ AOS and their derivatives can delay the growth of tumors and reduce tumor size. AOS may increase regulatory effects on the immune organs, such as spleen. ${ }^{26}$

There are some limitations for the present systematic review: 1) prospective randomized controlled trials were lacking; 2) the sample size was limited with the available number of osteoporosis patients and the small number of cases; a larger sample size is needed to improve statistical performance; 3 ) homogeneity of the complications was lacking and should be investigated in the future, especially in the study of nonrandomized controlled trials and in a large multicenter randomized controlled study; and 4) solubility of nanomaterials in water is important, to avoid immune clearance in human hosts. Low-molecular-weight AOS are cationic and highly soluble, although their solubility is limited by viscosity when the concentration is $>1 \%(\mathrm{v} / \mathrm{v})$. High-molecular-weight AOS are insoluble, although they may have a better activity. Improving the solubility of highmolecular-weight AOS will be the main work in the future.

\section{Conclusion}

PLIFC had higher fusion rate and JOA scores with ONAS administration. AOS had better improvement in complication rates than a PG group. AOS provide a better option for DLD therapy. AOS have been shown to be superior to normal nanoparticles by reducing miR-155, which affects antioxidant and anti-inflammatory properties of DLD patients. Further work is still needed to confirm the present results in the future.

\section{Disclosure}

The authors report no conflicts of interest in this work.

\section{References}

1. Donnarumma P, Tarantino R, Nigro L, et al. Decompression versus decompression and fusion for degenerative lumbar stenosis: analysis of the factors influencing the outcome of back pain and disability. J Spine Surg. 2016;2(1):52-58.

2. Guo S, Zeng C, Yan M, et al. A biomechanical stability study of extraforaminal lumbar interbody fusion on the cadaveric lumbar spine specimens. PLoS One. 2016;11(12):e0168498.

3. Barbanti Brodano G, Griffoni C, Zanotti B, et al. A post-market surveillance analysis of the safety of hydroxyapatite-derived products as bone graft extenders or substitutes for spine fusion. Eur Rev Med Pharmacol Sci. 2015;19(19):3548-3555.

4. Hodgson AR, Stock FE. Anterior spinal fusion - a preliminary communication on the radical treatment of Pott's disease and Pott's paraplegia (Reprinted from Clin Orthop Relat Res. 1956;44:266-275). Clin Orthop Relat Res. 2006;444:10-15.

5. Elias WJ, Simmons NE, Kaptain GJ, Chadduck JB, Whitehill R. Complications of posterior lumbar interbody fusion when using a titanium threaded cage device. J Neurosurg. 2000;93(1):45-52.

6. Sellimi S, Younes I, Ayed HB, et al. Structural, physicochemical and antioxidant properties of sodium alginate isolated from a Tunisian brown seaweed. Int J Biol Macromol. 2015;72:1358-1367.

7. Sen M. Effects of molecular weight and ratio of guluronic acid to mannuronic acid on the antioxidant properties of sodium alginate fractions prepared by radiation-induced degradation. Appl Radiat Isot. 2011;69(1):126-129.

8. Nguyen VT, Ko SC, Oh GW, et al. Anti-inflammatory effects of sodium alginate/gelatine porous scaffolds merged with fucoidan in murine microglial BV2 cells. Int J Biol Macromol. 2016;93(pt B): 1620-1632.

9. Igarashi A, Kikuchi S, Konno S. Correlation between inflammatory cytokines released from the lumbar facet joint tissue and symptoms in degenerative lumbar spinal disorders. J Orthop Sci. 2007; 12(2):154-160.

10. Chen $\mathrm{K}, \mathrm{Lv} \mathrm{X}, \mathrm{Li} \mathrm{W}$, et al. Autophagy is a protective response to the oxidative damage to endplate chondrocytes in intervertebral disc: implications for the treatment of degenerative lumbar disc. Oxid Med Cell Longev. 2017;2017:4041768.

11. Llacua A, de Haan BJ, Smink SA, de Vos P. Extracellular matrix components supporting human islet function in alginate-based immunoprotective microcapsules for treatment of diabetes. J Biomed Mater Res A. 2016;104(7):1788-1796.

12. Yin $\mathrm{N}$, Han $\mathrm{Y}, \mathrm{Xu} \mathrm{H}$, et al. VEGF-conjugated alginate hydrogel prompt angiogenesis and improve pancreatic islet engraftment and function in type 1 diabetes. Mater Sci Eng C Mater Biol Appl. 2016;59: 958-964.

13. Yuan K, Zhang X, Lv L, Zhang J, Liang W, Wang P. Fine-tuning the expression of microRNA-155 controls acetaminophen-induced liver inflammation. Int Immunopharmacol. 2016;40:339-346.

14. Eisenhardt SU, Weiss JB, Smolka C, et al. MicroRNA-155 aggravates ischemia-reperfusion injury by modulation of inflammatory cell recruitment and the respiratory oxidative burst. Basic Res Cardiol. 2015;110(3):32.

15. Yue MM, Gong WW, Qiao Y, Ding H. A method for efficient expression of Pseudomonas aeruginosa alginate lyase in Pichia pastoris. Prep Biochem Biotechnol. 2016;46(2):165-170. 
16. van den Eerenbeemt KD, Ostelo RW, van Royen BJ, Peul WC, van Tulder MW. Total disc replacement surgery for symptomatic degenerative lumbar disc disease: a systematic review of the literature. Eur Spine J. 2010;19(8):1262-1280.

17. Morgan JP, Miller AL, Thompson PA, Asfora WT. The Asfora Bullet Cage System shows comparable fusion rate success versus control cage in posterior lumbar interbody fusion in a randomized clinical trial. S D Med. 2016;69(4):157-165,167.

18. Choi MK, Kim SB, Park CK, Malla HP, Kim SM. Cross-sectional area of the lumbar spine trunk muscle and posterior lumbar interbody fusion rate: a retrospective study. Clin Spine Surg. 2017;30(6): E798-E803.

19. Tzankova V, Aluani D, Kondeva-Burdina M, et al. Hepatoprotective and antioxidant activity of quercetin loaded chitosan/alginate particles in vitro and in vivo in a model of paracetamol-induced toxicity. Biomed Pharmacother. 2017;92:569-579.

20. Aluani D, Tzankova V, Kondeva-Burdina M, et al. Cyrillicvaluation of biocompatibility and antioxidant efficiency of chitosan-alginate nanoparticles loaded with quercetin. Int J Biol Macromol. 2017; 103:771-782.
21. Hulsmans M, De Keyzer D, Holvoet P. MicroRNAs regulating oxidative stress and inflammation in relation to obesity and atherosclerosis. FASEB J. 2011;25(8):2515-2527.

22. Yang Z, Zheng B, Zhang Y, et al. miR-155-dependent regulation of mammalian sterile 20-like kinase 2 (MST2) coordinates inflammation, oxidative stress and proliferation in vascular smooth muscle cells. Biochim Biophys Acta. 2015;1852(7):1477-1489.

23. Zhang H, Zhao Z, Pang X, et al. Genistein protects against Ox-LDLinduced inflammation through MicroRNA-155/SOCS1-mediated repression of NF-kB signaling pathway in HUVECs. Inflammation. 2017;40(4):1450-1459.

24. Zhao R, Dong R, Yang Y, et al. MicroRNA-155 modulates bile duct inflammation via targeting suppressor of cytokine signaling 1 in biliary atresia. Pediatr Res. Epub 2017 Sep 6.

25. Batrakova EV, Kabanov AV. Pluronic block copolymers: evolution of drug delivery concept from inert nanocarriers to biological response modifiers. J Control Release. 2008;130(2):98-106.

26. Hu X, Jiang X, Hwang H, Liu S, Guan H. Antitumour activities of alginate-derived oligosaccharides and their sulphated substitution derivatives. Eur J Phycol. 2004;39(1):67-71.
International Journal of Nanomedicine

\section{Publish your work in this journal}

The International Journal of Nanomedicine is an international, peerreviewed journal focusing on the application of nanotechnology in diagnostics, therapeutics, and drug delivery systems throughou the biomedical field. This journal is indexed on PubMed Central,

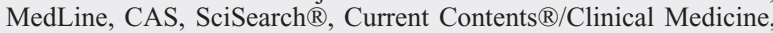

\section{Dovepress}

Journal Citation Reports/Science Edition, EMBase, Scopus and the Elsevier Bibliographic databases. The manuscript management system is completely online and includes a very quick and fair peer-review system, which is all easy to use. Visit http://www.dovepress.com/ testimonials.php to read real quotes from published authors. 